\title{
Ce que donne à entendre le mot médiation
}

\section{Nicole van der Elst}

\section{OpenEdition}

Journals

Édition électronique

URL : http://journals.openedition.org/communicationorganisation/2530

DOI : 10.4000/communicationorganisation. 2530

ISSN : 1775-3546

\section{Éditeur}

Presses universitaires de Bordeaux

\section{Édition imprimée}

Date de publication : 1 mai 2001

ISSN : 1168-5549

\section{Référence électronique}

Nicole van der Elst, "Ce que donne à entendre le mot médiation », Communication et organisation [En ligne], 19 | 2001, mis en ligne le 27 mars 2012, consulté le 04 mai 2019. URL : http://

journals.openedition.org/communicationorganisation/2530;DOI : 10.4000/ communicationorganisation. 2530

Ce document a été généré automatiquement le 4 mai 2019.

(c) Presses universitaires de Bordeaux 


\title{
Ce que donne à entendre le mot médiation
}

\author{
Nicole van der Elst
}

1 «Qu'entendez-vous par le mot de médiation?»

2 Posée l'an dernier à un public cultivé, lors d'un séminaire de recherche, cette question a suscité une trentaine de réponses, révélant à quel point cette fonction était encore méconnue dans notre pays. «Des médiateurs, je sais qu'il y en a un peu partout sur l'hexagone et de toutes les sortes, mais je ne situe pas très bien leurs attributions respectives. " Ainsi peut-on résumer l'état d'esprit dominant dans notre panel, au sein duquel le mot de médiation évoque le plus souvent des fantasmes positifs, souvent très anciens, telle cette image de Saint-Louis rendant la justice sous un chêne, qui a resurgi chez l'une de mes interlocutrices, actuellement conseil d'entreprise !

\section{Un cahier des charges flou, quelques personnalités émergentes}

3 Si son cahier des charges demeure flou, le suzerain de ce domaine est identifié sans ambiguïté par l'échantillon : c'est le Médiateur de la République, poste de haute portée symbolique, dont le titulaire émerge cependant difficilement de l'anonymat. Les conciliateurs, notables bénévoles, le plus souvent retraités, sont mieux identifiés, sans doute en raison du fait qu'ils disposent d'un lieu d'accueil accessible, au siège même des mairies.

4 Autres figures de prestige : les médiateurs de l'éducation nationale, ainsi qu'en témoigne cette appréciation : «le médiateur haut fonctionnaire, c'est quelqu'un qui n'a plus rien à prouver et qui n'est pas dans la course aux honneurs. C'est ce qui fonde sa crédibilité et son désintéressement. »

5 Un coup de chapeau est aussi accordé au médiateur du journal Le Monde, de la part de lecteurs fidèles, qui apprécient cette initiative. 


\section{La trêve de Dieu, précédent mémorable}

6 « Pour moi, la médiation, c'est ce qui permet à des esprits échauffés de surseoir à leurs intentions belliqueuses. » estime la doyenne de mon panel, ancien professeur d'histoire. Et de m'apprendre qu'au xième siècle, les évêques français avaient imposé à leurs ouailles une "paix de Dieu». faisant obligation de respecter un délai de quarante jours entre toute déclaration de guerre et commencement d'hostilités. La plupart des conflits sanguinaires importants étaient ainsi étouffés dans l'œuf, tandis que les chevaliers avaient obligation de déposer les armes plusieurs jours par semaine. Et cette érudite de conclure : « Les médiateurs ont aujourd'hui du pain sur la planche, » faisant ainsi allusion aux intervenants civils de paix et autres médiateurs sur les lieux conflictuels de la Planète.

\section{Un charisme qui ne se révèle qu'aux initiés}

7 La présente étude confirme ce que nous apprennent les pédagogues de cette discipline, à savoir que, pour apprécier en vérité le charisme du médiateur, il faut en avoir bénéficié en situation de "médié». Écoutons à ce propos le témoignage de J.X., peintre et philosophe, expulsée abusivement par un propriétaire : «la médiation, c'est une parole qui peut circuler entre opposants et va débloquer une situation sans issue. C'est une écoute qui prend en considération le point de vue de chacune des parties. Grâce à cette bouffée d'air pur, après des mois de procédures contre une institution brutale, ma cause est enfin apparue comme défendable et j'ai obtenu satisfaction: droit de retour, après rénovation, dans l'atelier que j'occupais depuis trente ans. Mon avocate n'avait rien pu obtenir. » Ainsi s'explique que l'initié ne confondra plus le charisme du médiateur avec celui, comparativement inefficace, du discours bien intentionné. Ce que reconnaît ce propos d'une visiteuse des prisons, n'ayant jamais bénéficié d'une formation de médiateur et s'avouant «ne pas savoir adresser à un futur délinquant la parole qui lui éviterait de commettre une bêtise.»

Une autre erreur, tout aussi fréquente, est due à une ignorance de l'impartialité requise du médiateur, vis-à-vis des deux adversaires du conflit où il intervient. Erreur qui conduit à confondre le rôle de médiateur avec celui d'un "chevalier blanc", comme dans cette affirmation : « Le médiateur est là pour défendre le faible, il est le bouclier du citoyen face à plus fort que lui l'institution, l'entreprise prêtes à le piétiner. "

Or l'analyse du rapport de forces ainsi évoqué, surtout en ce qui concerne le secteur marchand, demande à être nuancée. L'entreprise bien gérée a toujours intérêt à recourir au médiateur en temps utile, ne serait-ce que pour s'éviter la lourdeur du coût de gestion de tout conflit interminable avec un client récalcitrant. Ce que m'a confirmé Serge Bougaëff, médiateur attitré d'une caisse régionale bancaire, qui le charge d'élucider pour elle quelque deux mille dossiers par an, en accord avec les intéressés. "Il n'en reste pas moins, » observe-t-il, « que, lorsque l'on interroge ces derniers sur l'aspect le plus positif à leurs yeux de la médiation, ils vous répondent toujours : c'est le fait, pour la première fois depuis le début de mon litige, d'avoir enfin été écouté par quelqu'un. » 


\section{Vers un partage équitable du droit de parole?}

10 Une certaine désinformation est également perceptible concernant la déontologie que doit observer l'avocat dans le champ de la médiation, où il est désormais autorisé à agir non seulement en qualité de défenseur d'un médié, mais aussi en qualité de médiateur entre deux parties adverses.

11 Quand on sait que leurs traditions premières encourageaient les avocats à défendre prioritairement les faibles, - d'où peut-être la persistance du « chevalier blanc » dans la mémoire collective? - on peut se poser la question suivante: en instituant la qualification d'avocat-médiateur, le Barreau a t-il l'objectif d'effectuer un retour à ses sources ou simplement celui de contribuer, plus ou moins lucrativement, au désencombrement de la Justice? La question reste ouverte.

Dans toutes les hypothèses, le fait que sont aujourd'hui remis en cause, dans le sens d'une grandissante égalité, les rapports de force entre «notables-détenteurs de la parole » et « citoyens privés de parole » dans nos Démocraties permet d'éclairer le débat ci-dessus. Et autorise la médiation à être reconnue comme une forme privilégiée de «responsabilisation du citoyen", ainsi que s'en est félicité l'un des universitaires de notre panel.

\section{Le médiateur et son image}

13 Serviteur de la parole entre autrui et autrui, le médiateur propose une écoute accueillante à tous les usagers de l'espace public. Ce statut de fait lui permet, sans attendre d'autre reconnaissance que celle de ses concitoyens, de bénéficier, la plupart du temps, de l'image positive qu'ils accordent aux autres acteurs de la solidarité nationale.

14 La présente enquête, qui demanderait à être élargie pour une confirmation à cet égard, permet de discerner, au sein de l'opinion, des courants susceptibles de conforter les médiateurs dans la dynamique dont ils se disent porteurs. Une tâche qui exige de leur part - comme chez nombre d'autres innovateurs sociaux - une synergie concertée avec des interlocuteurs que, semble-t-il, ils n'ont pas encore mobilisés à ce jour. Parmi les éléments de cet actif dormant, figurent les personnes ayant découvert, grâce à l'intervention du médiateur, des solutions d'apaisement à leurs conflits. Public réceptif et influent dans toutes les classes de la Société et dont il conviendrait de ne pas laisser s'évaporer dans l'oubli le témoignage.

Une deuxième catégorie de public paraît également digne de plus grande attention de la part des médiateurs et cela, bien évidemment, dans un tout autre esprit que celui du marketing utilitariste. Elle englobe ces victimes d'agressions, qui ne se croyant pas assez fortes pour supporter les tensions inhérentes à la liquidation de leurs conflits, se trouvent entrainées, faute de culture adéquate, à fuir la médiation, qu'elles confondent avec une psychanalyse sauvage! Attitude qui m'a été résumée en ces termes par une praticienne libérale: «quand le dialogue n'est plus possible avec le client, j'agis de façon dure et prompte. Je m'en vais, même s'il doit m'en coûter financièrement. Je ne supporte pas qu'on me fasse souffrir avec des discussions pénibles. »

16 Il est clair que l'imprégnation, actuellement belliciste, de notre société ne facilite pas sa reconversion en un milieu réceptif aux méthodes de résolution non violente des conflits. 
À cet égard, le développement récent des pratiques de la «médiation par les pairs » en milieu scolaire constitue, à terme, une initiative très favorable au développement de la culture de paix. Encore faudrait-il qu'elle trouve à se généraliser à tous les âges de la vie pour qu'elle puisse porter pleinement ses fruits.

\section{RÉSUMÉS}

Alors que le recours à la médiation rencontre aujourd'hui une certaine audience dans notre pays, le mode d'emploi de cet outil de règlement des conflits demeure encore largement méconnu de l'opinion. D'où la persistance de nombreux fantasmes, que la présente recherche s'efforce d'élucider car ils menacent fortement son développement.

At the time when mediation is in the process of becoming more popular in our country, the ways to use this conflict-solving tool are still misunderstood of the larger audience. Hence many fantasies that this research paper aims at elucidating, thince it may threaten its development.

\section{INDEX}

Mots-clés : médiation, société, méconnaissance

\section{AUTEUR}

\section{NICOLE VAN DER ELST}

Nicole van der Elst a été responsable en entreprise de relations publiques institutionnelles (1965-1978), puis consultante dans un Cabinet-Conseil en mesure d'opinion (1979-1984). Elle a enseigné au CELSA(1970-1977) et à l'ISIT (Institut catholique de Paris), en qualité de chef de département. (1978-1992). Rédactrice en chef de la Maison de Verre, (1980-1982) elle a été journaliste à la revue Entreprises-Formation (1978-1994). Aujourd'hui consultante en milieu associatif, elle a publié récemment plusieurs essais dans le domaine de l'innovation sociale, notamment à la revue Humanisme et Entreprise. 